\title{
A Calculadora Financeira HP-12C como ferramenta para o Ensino de Estatística em um curso de Administração
}

Juliano Schimiguel

Josney Freitas Silva

\begin{abstract}
Resumo
Este trabalho apresenta um recorte da pesquisa desenvolvida durante o curso de mestrado e que possui envolvimento na pesquisa que está sendo desenvolvida pelo primeiro autor durante o curso de doutorado. Trata-se de uma investigação realizada no Ensino Superior sobre Educação Estatística e o uso de recursos tecnológicos, neste caso, da Calculadora Financeira HP-12C. A investigação se desenvolveu em duas turmas do curso de Administração em uma universidade pública do Estado de Minas Gerais e teve como objetivo utilizar a Calculadora Financeira HP-12C no desenvolvimento da Educação Estatística no Ensino Superior. A metodologia utilizada baseou-se na pesquisa-ação e os instrumentos de coleta de dados constituíram-se dos protocolos dos alunos em dois momentos distintos, um Simulado em grupo e uma Prova individual. O referencial teórico aporta-se em Albergaria e Ponte (2008), Melo (2008), Guinther (2009) quanto à utilização de calculadoras no ensino; Ponte, Brocardo e Oliveira (2009), Silva (2014), Campos, Wodewotzki e Jacobini (2011) quanto a Educação Estatística, entre outros. Os resultados apresentados são satisfatórios em relação à mobilização dos conhecimentos apresentados pelos estudantes principalmente quanto à interpretação dos resultados obtidos na resolução dos exercícios.
\end{abstract}

Palavras-chave: Ensino Superior, Educação Estatística, Calculadora Financeira HP-12C.

\section{Abstract}

This paper presents part of the research developed during the master course and has involvement in research being developed by the first author during the PhD program. This is an investigation in Higher Education on Statistics Education and the use of technological resources, in this case, the HP-12C Financial Calculator. The research was developed in two classes of Business Administration course at a public university in the state of Minas Gerais and aimed to use the HP-12C Financial Calculator in the development of Statistics Education in Higher Education. The methodology used was based on action research and the data collection instruments consisted of the records of students at two different times, one Simulated group and individual Test. The theoretical framework brings in Albergaria and Ponte (2008), Melo (2008), Guinther (2009) on the use of calculators in teaching; Ponte, Brocardo and Oliveira (2009), Silva (2014), Campos, Wodewotzki and Jacobini (2011) as the Statistics Education, among others. The results are satisfactory regarding the mobilization of knowledge presented by the students especially regarding the interpretation of results in solving the exercises.

Keywords: Higher Education, Statistics Education, HP-12C Financial

DOI: Em andamento. 


\section{Introdução}

O auxílio de calculadoras na resolução de cálculos matemáticos, em geral, proporciona facilidade e eficiência na solução, principalmente naqueles casos mais complexos ou que envolvem números de grandeza considerável. Entretanto, o uso de calculadoras, em sala de aula, sempre enfrentou uma considerável resistência dos docentes, por estes entenderem que sua utilização seria prejudicial ao desenvolvimento cognitivo dos estudantes.

Tal temática justifica-se, uma vez que há indícios que apontam para o fato de que, no âmbito do Curso Superior em Administração, além de resistentes ao progresso, os docentes ignoravam as próprias Diretrizes Curriculares do Curso que, em seu teor, incentivam o uso de recursos tecnológicos (BRASIL, 2005) e também desconhecem que diversos pesquisadores, em diferentes países, apresentam resultados interessantes quanto à utilização de calculadoras, nos diferentes níveis de escolarização, conforme apontam Albergaria e Ponte (2008), Melo (2008), Guinther (2009), entre outros.

A Calculadora Financeira HP-12C pode ser utilizada para realizar alguns cálculos estatísticos para uma ou duas variáveis, como, por exemplo, a média, o desvio padrão, a estimação linear e a média ponderada, bem como o coeficiente de correlação entre duas variáveis, embora seja altamente capacitada para efetuar cálculos provenientes da área financeira. Deste modo, o objetivo deste trabalho é apresentar como esta calculadora pode ser utilizada no ensino de Estatística e aumentar sua potencialidade de uso, bem como sua popularidade entre os acadêmicos dos cursos de Administração, Economia, Ciências Contábeis e demais cursos correlatos.

Uma vez que sua utilização em cálculos financeiros já é bem popular, ressalta-se a importância de se conhecer o uso das funcionalidades estatísticas da Calculadora Financeira HP12C, salientando que pouco se discute quanto a sua utilização em cálculos estatísticos, os quais, muitas vezes são efetuados por meio de outros instrumentos, como as calculadoras científicas.

Deste modo, nos pautamos nos preceitos da Educação Estatística, à luz das obras de Silva (2014), Campos, Wodewotzki e Jacobini (2011) e Ponte, Brocardo e Oliveira (2009) que defendem a corrente de que a utilização de recursos tecnológicos como auxílio à resolução de cálculos algébricos costumeiramente complexos deve ser aplicada de modo a permitir aos estudantes sua dedicação à análise e interpretação dos resultados obtidos por tal resolução, objeto que consideramos como o verdadeiro motivo que leva os estudantes a se enveredarem pela busca de resultados dos problemas a eles propostos.

Este trabalho está organizado em cinco seções, sendo que a primeira, nomeada como introdução, apresenta a temática a ser discutida, a justificativa e os objetivos, bem como o 
referencial teórico e a estrutura do texto. A segunda seção traz o referencial teórico ao qual este trabalho se fundamenta e aborda o uso da Calculadora Financeira e sua relação com a Educação Estatística. A metodologia de pesquisa adotada é apresentada na terceira seção, juntamente com a descrição dos instrumentos de coleta dos dados e a análise da utilização da Calculadora Financeira HP-12C no Ensino de Estatística é tema da quarta seção deste trabalho. A quinta seção apresenta as considerações finais, sendo seguida pelos agradecimentos e as referências.

\section{Uso da Calculadora Financeira e a Educação Estatística}

Albergaria e Ponte (2008) apresentam que até pouco tempo atrás, havia apenas o uso dos algoritmos escritos como processo de cálculos e, por conseguinte, o meio de ensino nas escolas era baseado nestes algoritmos.

Não obstante, somos obrigados a repensar esta situação o surgimento das tecnologias, pois, conforme apresentam Albergaria e Ponte (2008, p. 02), "a forma como o sentido do número se expressa varia segundo diferentes dimensões da atividade matemática". Deste modo, percebemos que a opção por um processo de cálculo adequado depende do grau de complexidade de desenvolvimento do sentido do número, além da correta interpretação e utilização dos resultados provenientes obtidos por meio de quaisquer métodos utilizados.

A utilização dos algoritmos escritos, consequentemente, assume uma nova perspectiva com o surgimento da calculadora e do computador, que passam a ser empregados para cálculos com números de grandeza elevada, com muitos dígitos ou muitas casas decimais. A capacidade de realizar cálculos mentais é essencial a todos no dia-a-dia. O que nos remete a importância de lembrar, a este respeito, que o uso de estratégias e referências pessoais se traduz por meio da utilização de números com sentido.

Albergaria e Ponte (2008, p. 05) também apresentam que o uso da calculadora possibilita como um de seus benefícios "o acesso à resolução de tarefas exploratórias ou de investigação a alunos com dificuldade na utilização de algoritmos escritos". Assim, oportunidade de desenvolver o raciocínio matemático dos alunos é alcançada com o uso da calculadora, por meio da identificação de propriedades numéricas, possibilitando-Ihes determinar padrões numéricos mediante situações trabalhadas e a estabelecer generalizações.

O uso da calculadora em sala de aula é pauta daqueles que se dedicam à Educação Matemática, que debatem e dedicam a atenção com maior visibilidade, conforme demonstra Guinther (2009) ao afirmar que Malba Tahan, em 1961, na obra Didática da Matemática, já abordava o uso de máquinas de calcular para resolver cálculos mais trabalhosos e intrincados.

De acordo com Guinther (2009), o alto custo configurava como o argumento para não se utilizar a calculadora no ensino, uma vez que essa máquina era praticamente inacessível aos 
estudantes em geral. Além do que, conforme também relata Melo (2008), se acreditava que o uso da calculadora em sala de aula deixaria os estudantes preguiçosos e que estes desaprenderiam os algoritmos e deixariam de raciocinar.

Aqueles que possuem conhecimento e são adeptos à tecnologia encontram-se distantes, por conta destas crenças, daqueles que foram privados, na escola, do acesso a esta tecnologia. Concordamos então com Guinther (2009, p. 60) ao afirmar que "de nada adianta uma máquina nas mãos de uma pessoa que não sabe quais cálculos deve efetuar". Isto porque para resolver determinadas tarefas em um dado contexto, a régua e o compasso, assim como a calculadora constituem ferramentas que podem ser utilizadas nas mais diversas situações. Entretanto, o resultado não será satisfatório caso instruções equivocadas forem introduzidas na calculadora. Logo, saber interpretar os números e identificar qual operação deve-se efetuar torna-se imprescindível e necessário.

O professor pode trabalhar com situações numéricas que só seriam possíveis com a utilização da calculadora, conforme aponta Melo (2008), evitando problemas causados pela utilização de cálculos que recaiam em resultados que não sejam exatos, momento em que tais cálculos têm sua veracidade questionada por muitos estudantes.

O nosso objeto de estudo vai de encontro ao pensamento de Melo (2008), quando declara que muitos profissionais, ao se utilizarem de calculadoras ou de outro tipo de tecnologia para executarem suas atividades, aproveitam e desfrutam, ao máximo, dos benefícios por elas oferecidos, mas que, entretanto, na Educação, o preconceito pelo uso deste instrumento primordial e fundamental em nossa era, a da tecnologia, ainda deve ser enfrentado e desmistificado.

Por outro lado, desde o século XX, mais especificamente na década de 90, as origens das dificuldades pedagógicas dos estudantes em relação à "Estatística" têm sido o incentivo da busca inúmeros pesquisadores, momento em que aparecem as investigações envolvendo o ensino e a aprendizagem nesta área, culminando na formulação de uma nova área de atuação pedagógica, a Educação Estatística, conforme apresenta Silva (2014).

Segundo Campos, Wodewotzki e Jacobini (2011), a ocorrência de três competências que devem estar presentes no planejamento de cursos de "Estatística" (a literacia estatística, o raciocínio estatístico e o pensamento estatístico), as quais são indispensáveis ao aprendizado dos conceitos fundamentais desta disciplina. Noutros termos, diz-se que a habilidade de ler, compreender, interpretar, analisar e avaliar textos escritos referentes à "Estatística" é considerada por Campos, Wodewotzki e Jacobini (2011) como literacia estatística. Ou seja, à habilidade de argumentar usando a terminologia estatística de forma correta, sendo ainda capaz de organizar dados, construir e apresentar tabelas e trabalhar com diferentes representações dos dados como tabelas e gráficos. O uso de tecnologia nos cálculos é incentivado pelos autores, 
portanto, para valorizar as interpretações dos resultados. Neste sentido, os autores alertam para a importância dos estudantes tomarem as rédeas de seu próprio aprendizado, tendo a oportunidade de que estes produzam seus próprios dados e que encontrem os resultados básicos.

Nesta linha de pensamento, e apesar de inserida num contexto de divergências conceituais que, aliás, é próprio do fazer científico, concordamos com Ponte, Brocardo e Oliveira (2009, p. 105) ao defenderem, assim como muitos outros autores, que a Educação Estatística deve se deslocar do campo do cálculo e da realização de tarefas para o processo de investigação.

Ponte, Brocardo e Oliveira citam o estudioso e estatístico Richard Snee, ao apontar como ênfases da Educação Estatística a "recolha de dados, a compreensão e a modelação da variação, representação gráfica de dados, experimentação, questionamento", priorizando assim o "modo como o pensamento estatístico é usado na investigação de problemas do mundo real" (SNEE, 1993, p. 151, apud PONTE; BROCARDO; OLIVEIRA, 2009, p. 105).

Como apresentado em Silva (2014), os estudantes passam a compreender o verdadeiro papel da "Estatística" em nossa sociedade por meio de como os conteúdos estatísticos podem ser desenvolvidos. Mais precisamente, o Ensino de Estatística pode se tornar uma atividade mais focada em aplicações específicas das respectivas áreas de conhecimento em questão.

\subsection{A Calculadora Financeira HP-12C}

A Calculadora Financeira HP-12C (Figura 1) constitui-se de uma ferramenta de grande utilização, especificamente, aos acadêmicos dos cursos de Administração e correlatos, pois entendemos a relação estreita que existe entre eles, uma vez que, aos profissionais que enveredam para a área financeira, a calculadora consiste em uma ferramenta de extrema importância (SILVA, 2014).

Quando nos deparamos com alguns cálculos que não são possíveis de serem realizados apenas com lápis e papel, seguindo algoritmos escritos, dado a complexidade de cálculos e grandeza de valores ou, por outro lado, pela utilização de índices infinitesimais, o uso da Calculadora Financeira HP-12C se faz necessário. Cálculos estes que são comuns àqueles acadêmicos que devem cursar as disciplinas de "Administração e Análise de Custos", "Gestão Financeira", "Gestão Orçamentária", "Contabilidade Gerencial", "Contabilidade Empresarial" e "Matemática Financeira". 


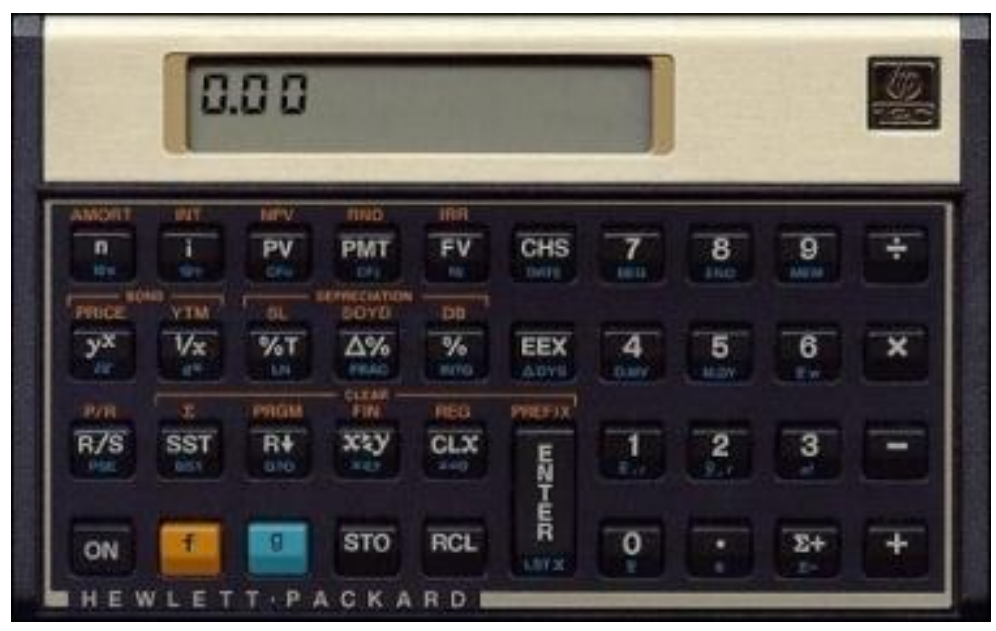

Figura 1 - Calculadora HP-12C.

(Fonte: Hewlett-Packard Company, 2008)

A Calculadora Financeira HP-12C, segundo Silva (2014), não realiza cálculos da mesma forma como as calculadoras científicas existentes no mercado, uma vez que ela utiliza a Notação Polonesa Inversa - RPN - Reverse Polish Notation (HEWLETT-PACKARD COMPANY, 2008) apresentando certa complexidade quanto a sua forma de operacionalização. Assim, sua utilização difere quanto às outras calculadoras financeiras ou científicas disponíveis no mercado, não sendo tão intuitiva.

\subsection{A estatística presente na Calculadora Financeira HP-12C}

A Calculadora Financeira HP-12C contempla cálculos estatísticos uni e bivariados. Assim, pode-se armazenar em sua memória um conjunto de dados por meio da tecla $[\Sigma+]$, que calcula e armazena estatísticas dos dados nos registros de armazenamento $R_{1}$ a $R_{6}$, denominados registros estatísticos automaticamente. Em cálculos estatísticos uni-variados, para cada dado, denominado "valor de $x$ ", digitamos o valor de $x$, seguido da tecla $[\Sigma+]$. Quando estamos trabalhando com cálculos bivariados, para cada par ordenado, denominado "valores de $\mathrm{x}$ e $\mathrm{y}$ ", digitamos inicialmente o valor de $y$, seguido da tecla "Enter", depois digitamos o valor de " $x$ ", seguido da tecla $[\Sigma+]$. Antes de se iniciar a introdução de um novo conjunto de dados, deve-se zerar os registradores estatísticos pressionando as teclas [f] CLEAR $[\Sigma]$.

As estatísticas acumuladas são armazenadas conforme apresentado no Quadro 1. 
Quadro 1 - Registros e Valor Estatístico na HP-12C.

\begin{tabular}{|l|l|}
\hline Registro & Valor Estatístico \\
\hline$R_{1}$ & $n:$ número de dados acumulados. \\
\hline$R_{2}$ & $\Sigma x:$ soma dos valores de $\mathrm{x}$. \\
\hline $\mathrm{R}_{3}$ & $\Sigma \mathrm{x}^{2}$ : soma dos quadrados dos valores de $\mathrm{x}$. \\
\hline $\mathrm{R}_{4}$ & $\Sigma \mathrm{y}$ : soma dos valores de $\mathrm{x}$. \\
\hline $\mathrm{R}_{5}$ & $\Sigma \mathrm{y}^{2}:$ soma dos quadrados dos valores de $\mathrm{y}$. \\
\hline $\mathrm{R}_{6}$ & $\Sigma \mathrm{xy}$ : soma dos produtos dos valores de $\mathrm{x}$ e valores de $\mathrm{y}$. \\
\hline
\end{tabular}

(Fonte: Hewlett-Packard Company, 2004)

É possível calcular a média aritmética, média ponderada, o desvio-padrão, o coeficiente de correlação e a estimação linear com a Calculadora Financeira HP-12C. Entretanto, poucos sabem como utilizar os recursos nela disponibilizados. Considerando seu potencial tanto em cálculos financeiros, motivo pelo qual esta calculadora é de grande utilidade, quanto em cálculos estatísticos, consideramos esta calculadora como uma excelente ferramenta aos Administradores e demais profissionais que trabalham com operações financeiras.

\section{Metodologia}

Com o intuito de verificar a utilização da Calculadora Financeira HP-12C no Ensino de Estatística, procedemos a uma investigação pautada na Pesquisa-ação, cujo cenário constitui-se do corpo discente do segundo período do curso de Administração, devidamente matriculado na disciplina de Métodos Quantitativos em Administração, em uma universidade pública do Estado de Minas Gerais, formado por duas turmas, sendo uma turma no período matutino e outra no período noturno, denominadas Turma A e Turma B, respectivamente. A população investigada (unidade de análise) consiste em 24 alunos no turno matutino e 42 alunos no turno noturno (alunos matriculados nesta disciplina que chegaram ao final do semestre letivo). O período de investigação corresponde ao intervalo de agosto a dezembro de 2012.

Segundo Moreira e Caleffe (2008, p. 91), na Pesquisa-ação "o enfoque é um problema específico em um cenário específico". Igualmente ao que os autores apresentam, não procuramos um "conhecimento generalizável, mas a um conhecimento preciso para um propósito e situação particulares".

O uso da pesquisa-ação em escola e em sala de aula é uma forma: 
"a) de sanar problemas diagnosticados em situações especificas, ou melhorar de alguma maneira um conjunto de circunstâncias; b) de treinamento em serviço, portanto, proporcionando ao professor novas habilidades, métodos para aprimorar sua capacidade analítica e o fortalecimento da autoconsciência; c) de introduzir abordagens adicionais e inovadoras no processo ensino-aprendizagem e aprender continuamente em um sistema que normalmente inibe a mudança e inovação." (MOREIRA \& CALEFFE, 2008, p. 92).

Deste modo, o uso da Pesquisa-ação é apropriado quando se deseja investigar sobre métodos de ensino, estratégias de aprendizagem, procedimentos de avaliação, atitudes e valores, desenvolvimento pessoal dos professores, gerenciamento e controle e gestão (MOREIRA \& CALEFFE, 2008, p. 93-94).

A disciplina em questão possui carga horária correspondente a oitenta horas, tendo as aulas ministradas em quatro horas semanais distribuídas em dois dias por semana. As avaliações, segundo as normas da universidade constituem-se de duas etapas, em que à primeira é atribuído um valor de quarenta $(40,00)$ pontos e à segunda, sessenta $(60,00)$ pontos, considerando que nenhuma das avaliações em cada etapa pode exceder a quarenta $(40,00)$ pontos.

Optamos por distribuir as avaliações em dois Simulados, duas Provas e o Projeto de Investigação Estatística. As provas foram realizadas individualmente, enquanto os simulados foram realizados em grupos de até quatro integrantes e o Projeto de Investigação Estatística em grupos de até seis integrantes. Assim, determinamos como instrumentos para coleta de dados para esta pesquisa uma questão que aparece tanto no Simulado II quanto na Prova II.

\section{Análise do uso da Calculadora Financeira HP-12C}

Para avaliar o aprendizado dos alunos ao final da disciplina, aplicamos uma avaliação em grupo, denominada Simulado II e uma avaliação individual, denominada Prova II conforme descrito na seção anterior. Nesta ultima, solicitamos aos alunos que indicassem qual tipo de calculadora utilizaram para a resolução dos exercícios.

Na Tabela 1, observamos que a utilização da calculadora HP-12C foi mais evidente na Turma B, onde $57,14 \%$ dos seus alunos a utilizaram, enquanto apenas $20,83 \%$ dos alunos da Turma A utilizaram esta calculadora. Quando observamos o total de alunos, percebemos que 51,52\% utilizaram a Calculadora Científica, 43,94\% utilizaram a Calculadora Financeira HP-12C enquanto $4,55 \%$ utilizaram calculadoras simples. 
Tabela 1 - Calculadoras utilizadas por Turma

\begin{tabular}{|c|c|c|c|c|c|c|}
\hline \multirow{2}{*}{ Calculadora } & \multicolumn{2}{|c|}{ Turma A } & \multicolumn{2}{|c|}{ Turma B } & \multicolumn{2}{|c|}{ Total } \\
\hline & $\mathrm{N}$ & $\%$ & $N$ & $\%$ & $\mathrm{~N}$ & $\%$ \\
\hline Científica & 19 & 79,17 & 15 & 35,71 & 34 & 51,52 \\
\hline HP-12C & 5 & 20,83 & 24 & 57,14 & 29 & 43,94 \\
\hline Simples & 0 & 0,00 & 3 & 7,14 & 3 & 4,55 \\
\hline Total & 24 & 100,00 & 42 & 100,00 & 66 & 100,00 \\
\hline
\end{tabular}

Fonte: Elaborado pelo autor.

Observando o Gráfico comparativo na Figura 2, percebemos a evidência de que a Turma A utilizou mais a Calculadora Científica, enquanto a Calculadora HP-12C foi a mais utilizada pela Turma B.

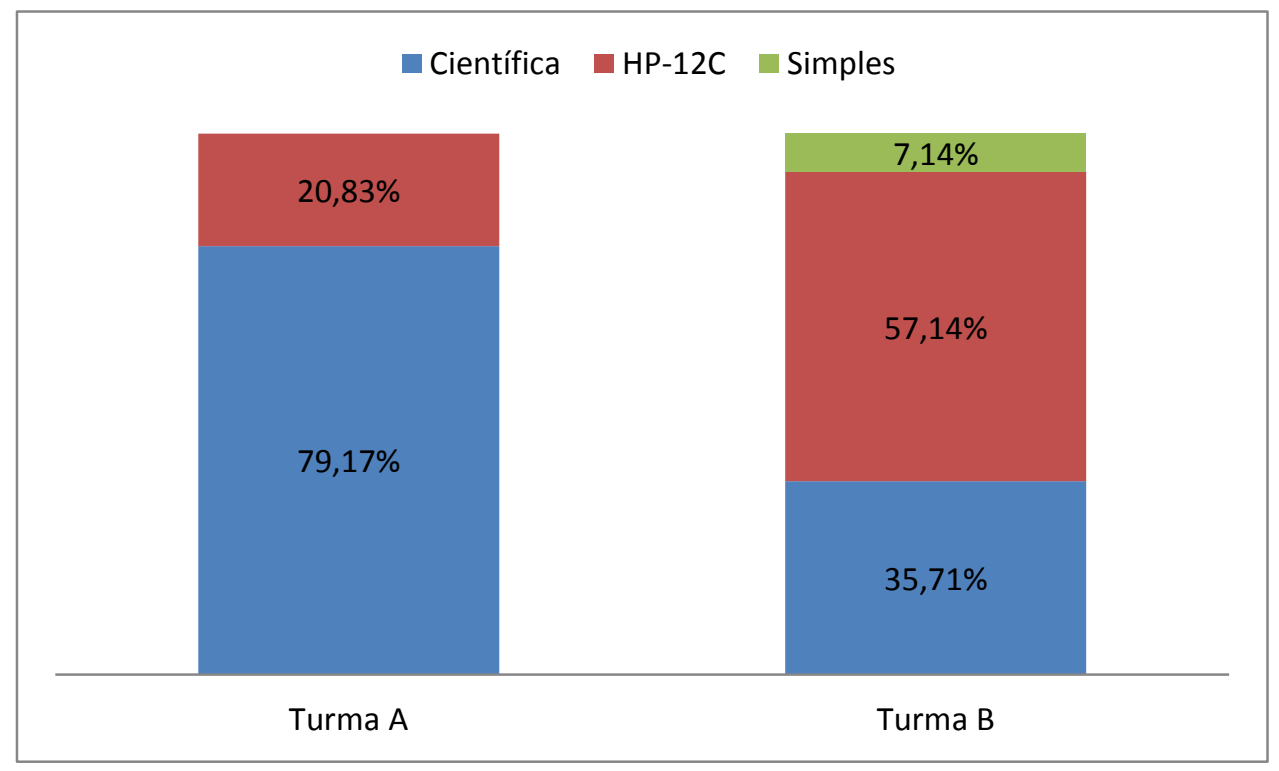

Figura 2- Gráfico do tipo de Calculadoras utilizadas por Turma (\%)

Ao proceder à análise das produções dos alunos, escolhemos por utilizar uma questão que aparecia tanto no Simulado II quanto na Prova II. Tal escolha se deu pela complexidade da questão, que envolve os conteúdos sobre cálculo de Média Aritmética, Variância, Desvio-padrão, Coeficiente de Variação, Covariância, Coeficiente de Correlação e Diagrama de Dispersão. A questão em cada uma das avaliações apresentavam enunciados distintos, porem, as exigências eram semelhantes. $O$ enunciado de uma delas foi exposto como a seguir:

“Cinco observações tomadas para duas variáveis são apresentadas a seguir: 


\begin{tabular}{l|lllll}
$X_{i}$ & 0 & 5 & 0 & 5 & 0 \\
\hline$Y_{i}$ & 8 & 4 & 0 & 6 & 2
\end{tabular}

a) Calcule e interprete a Covariância e o Coeficiente de Correlação da amostra.

b) Calcule o Coeficiente de Variação para as variáveis $\mathrm{X}$ e $\mathrm{Y}$.

Desenvolva um Diagrama de Dispersão para estas variáveis."

Analisando os protocolos individuais dos alunos, relativos à Prova II, podemos observar que, ao utilizar a Calculadora HP-12C e suas funções estatísticas, o conhecimento de conceitos estatísticos também se faz necessário, uma vez que a calculadora não oferece tudo que o aluno precisa. Porém, Ihe permite chegar onde precisa, fazendo uso de seu próprio conhecimento, conforme demonstrado pela Figura 3. Neste caso, o aluno, para encontrar a Covariância, utilizou o Desvio-padrão das duas variáveis e o Coeficiente de Correlação destas mesmas variáveis, consistindo no processo inverso geralmente utilizado, onde utilizamos a Covariância e o Desviopadrão das duas variáveis para se encontrar o Coeficiente de Correlação.

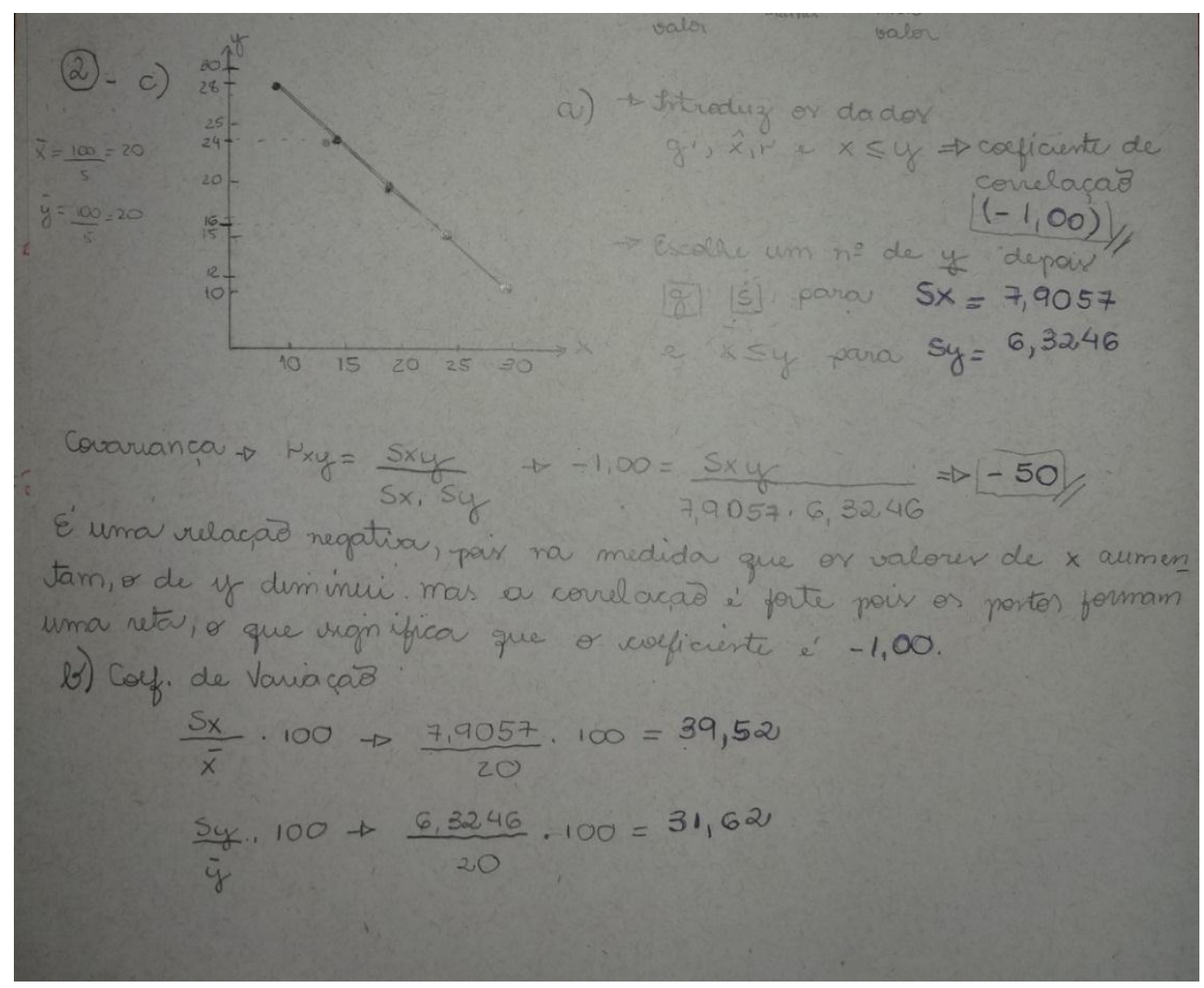

Figura 3 - Protocolo de um aluno que realizou a questão analisada com a Calculadora HP-12C utilizando suas funções. 
Também observamos protocolos onde os alunos, mesmo utilizando a Calculadora HP-12C, recorreram ao método de construção de uma tabela para cálculos auxiliares a fim de calcular a Variância, o Desvio-padrão, a Covariância e finalmente o Coeficiente de Correlação como apresentado na Figura 4. O procedimento, apesar de longo, possibilitou ao aluno, o manuseio dos dados, em sua totalidade. Por se tratar de uma avaliação em sala de aula, a questão foi elaborada com uma quantidade reduzida de observações, o que, tornou mais fácil a adoção deste método.

Ao trabalhar com um número maior de observações, a utilização dos recursos estatísticos da Calculadora Financeira HP-12C se faz mais eficiente, uma vez que não se faz necessário o uso de cálculos repetitivos. Outro fator que confere ao uso da Calculadora Financeira HP-12C maios eficiência está relacionado à minimização dos erros possivelmente cometidos por aproximações de casas decimais em cálculos intermediários, o que gera sensíveis diferenciações nos resultados finais.

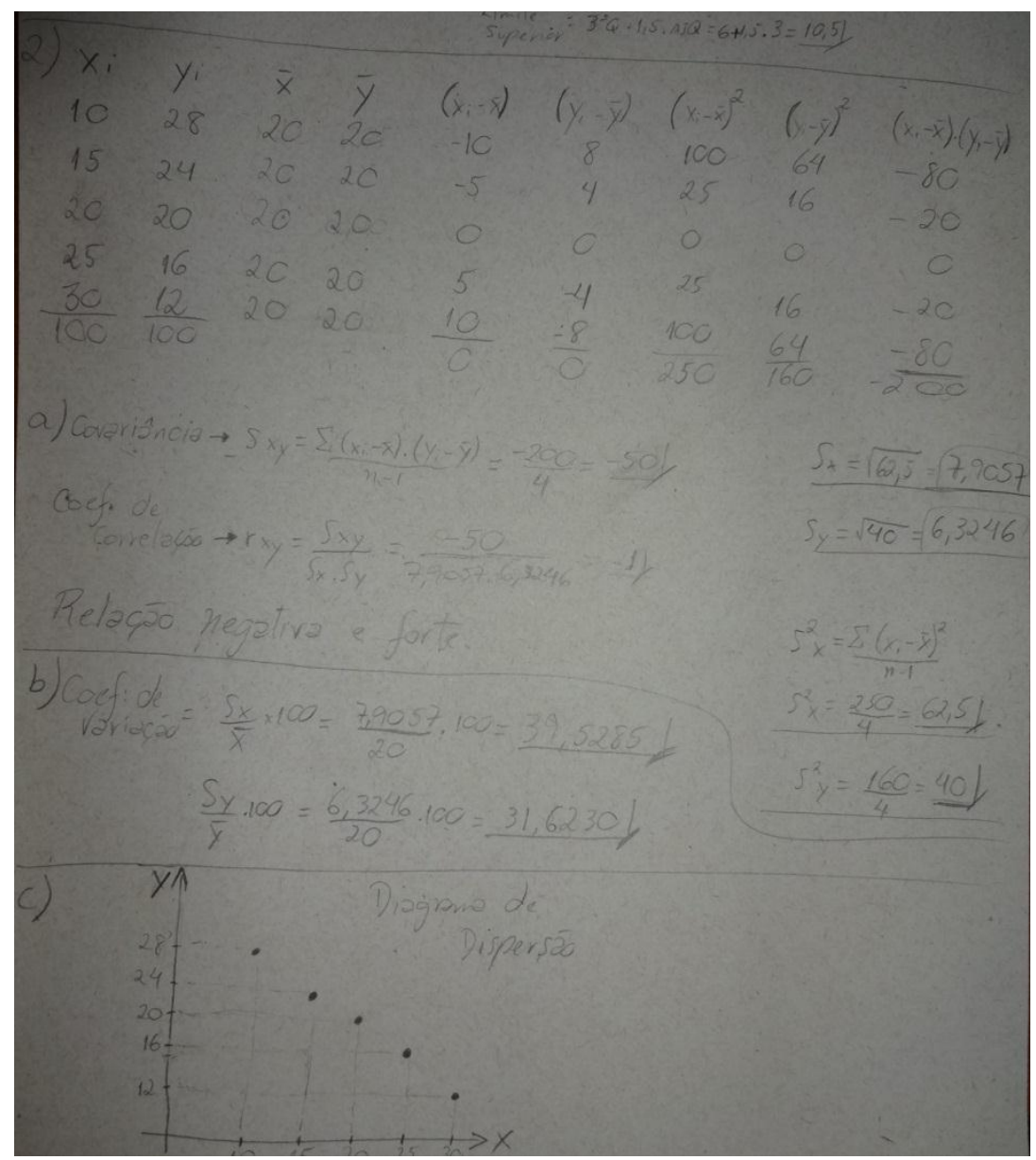

Figura 4 - Protocolo de um aluno que realizou a questão analisada com a Calculadora HP-12C sem utilizar suas funções.

Nestes dois exemplos apresentados por meio da Figura 3 e da Figura 4, podemos observar que o conhecimento matemático não sofreu prejuízo dado ao fato de que no primeiro caso, o 
discente fez o uso das funções estatísticas presentes na Calculadora Financeira HP-12C. Pelo contrário, o discente apresentou maior domínio dos conceitos estatísticos ao argumentar sobre a relação entre o resultado da covariância e do coeficiente de correlação, relacionando ainda ao resultado apresentado pelo diagrama de dispersão, fato este que corrobora com os preceitos da Educação Estatística, onde o que realmente importa é a interpretação dos resultados e não somente a perfeita utilização dos algoritmos para se chegar aos resultados.

\section{Considerações finais}

A Calculadora HP-12C é amplamente conhecida no mercado como uma grande ferramenta para profissionais da área financeira. Entretanto, ela também apresenta algumas ferramentas estatísticas que são muito utilizadas em diversas áreas do conhecimento. $\mathrm{O}$ uso desta calculadora em sala de aula contribui consideravelmente no ensino de Estatística, porque os estudantes podem realizar cálculos complexos de forma rápida, e se aterem às interpretações dos resultados obtidos por meio dos cálculos efetuados. Deste modo, dá-se mais atenção às interpretações dos dados que representam os números investigados, do que aos algoritmos complexos que levam os estudantes a se preocupar em chegar ao resultado final dos cálculos, sem a devida atenção ao real significado destes resultados.

A estratégia de se utilizar ferramentas tecnológicas como auxílio aos cálculos estatísticos, possibilitando uma maior ênfase na interpretação do significado dos dados investigados, está diretamente ligada aos conceitos de Educação Estatística. Assim, ao analisar os protocolos dos alunos aqui apresentados, percebemos a mobilização dos conteúdos estatísticos pelos estudantes e entendemos ser pertinente o uso desta ferramenta em sala de aula, porque permite aos estudantes, não só aprender a utilizar a Calculadora Financeira HP-12C para efetuar cálculos estatísticos como os apresentados neste trabalho, mas também desenvolverem maior senso crítico e de análise para interpretar os resultados obtidos por meio dos cálculos efetuados pela calculadora.

\section{Agradecimentos}

À FAPEMIG pela concessão de bolsa PC-RH para a realização do curso de Mestrado em Ensino de Ciências junto a Universidade Cruzeiro do Sul - SP (2012-2013) e à CAPES pela concessão de bolsa para a realização do Doutorado em Ensino de Ciências e Matemática junto a Universidade Cruzeiro do Sul - SP (2014).

\section{Referências}

ALBERGARIA, I. S.; PONTE, J. P. Cálculo mental e calculadora. In: CANAVARRO, A. P.; MOREIRA, D.; ROCHA, M. I. (Org.). Tecnologias e educação matemática. Lisboa: SEM-SPCE, 2008. p. 98-109. 
Disponível em: $\quad<$ http://www.educ.fc.ul.pt/docentes/iponte/docs-pt/08-Albergaria-ponte\%20 EIEM.pdf>. Acesso em: 12 jul. 2013.

BRASIL. Resolução no 4, de 13 de julho de 2005. Institui as diretrizes curriculares nacionais do curso de administração, bacharelado e dá outras providências. Diário Oficial da União. Brasília, DF, no 137, 19 jul. de 2005. Seção 01.

CAMPOS, C. R.; WODEWOTZKI, M. L. L.; JACOBINI, O, R. Educação estatística: teoria e prática em ambientes de modelagem matemática. Belo Horizonte: Autêntica, 2011.

GUINTHER, A. Análise do desempenho de alunos do ensino fundamental em jogos matemáticos: reflexões sobre o uso da calculadora nas aulas de matemática. 2009.182 f. Dissertação (Mestrado em Ensino de Matemática)-Pontifícia Universidade Católica de São Paulo, São Paulo, 2009.

HEWLETT-PACKARD DEVELOPMENT COMPANY. HP-12C Calculadora financeira: guia do usuário. San Diego: Hewlett-Packard Company, 2004.

. HP-12C Calculadora financeira: guia de inicialização rápida. San Diego: Hewlett-Packard Company, 2008.

MELO, A. R. F. A prática do professor de matemática permeada pela utilização da calculadora. 2008. 141 f. Dissertação (Mestrado em Ensino de Matemática)- Pontifícia Universidade Católica de São Paulo, São Paulo, 2008.

MOREIRA, H.; CALEFFE, L. G. Metodologia da pesquisa para o professor pesquisador. Rio de Janeiro: Lamparina, 2008.

PONTE, J. P.; BROCARDO, J.; OLIVEIRA, H. Investigações em estatística. In: Investigações matemáticas em sala de aula. Belo Horizonte: Autêntica, 2009.

SILVA, J. F. O uso das TICs no ensino superior e suas contribuições para a educação estatística. 2014. 115 f. Dissertação (Mestrado em Ensino de Ciências) - Universidade Cruzeiro do Sul, São Paulo, 2014.

Juliano Schimiguel - Doutor em Ciência da Computação - IC/UNICAMP. Instituto de Computação, Universidade Estadual de Campinas. Departamento de Sistemas de Informação. Professor do Programa de Doutorado/Mestrado em Ensino de Ciências e Matemática - Universidade Cruzeiro do Sul. E-mail: juliano.schimiguel@cruzeirodosul.edu.br

Josney Freitas Silva - Mestre em Ensino de Ciências - Programa de Pós-Graduação em Ensino de Ciências e Matemática da Universidade Cruzeiro do Sul. Chefe do Departamento de Ciências Humanas e professor da Universidade do Estado de Minas Gerais (UEMG) - Câmpus de Frutal/MG. E-mail: josneyf@yahoo.com.br

R. B. E. C. T., vol 8, Ed. Sinect, jan-abr.2015 ISSN - 1982-873X

DOI: Em andamento. 